\title{
PERCEPCIÓN DE LOS PACIENTES Y DEL PERSONAL DEL PROGRAMA DIABETIMSS SOBRE EL ESTILO DE VIDA Y EL CONTROL METABÓLICO
}

Ma. de Jesús Torres-Dueñas ${ }^{1 *}$, Araceli Vázquez-Hernández² ${ }^{2}$ Issa Gil-Alfaro ${ }^{3}$ y Manolo Cerdán-Galán ${ }^{4}$

1,2Especialista en Medicina Familiar, Hospital General de Zona con Medicina Familiar Número 28, Martínez de la Torre, Veracruz; 3Profesora Adjunta Residencia de Medicina Familiar Unidad de Medicina Familiar Número 66, Xalapa, Veracruz; 'Licenciado en Estadística Xalapa, Veracruz. México

RESUMEN: Objetivo general: Determinar la percepción de pacientes y personal del programa DiabetIMSS sobre los motivos por los que no se logra control metabólico ni mejoría en estilo de vida de pacientes con diabetes mellitus (DM) en la Unidad de Medicina Familiar (UMF) 66. Material y métodos: Estudio mixto en pacientes diabéticos y personal DiabetIMSS previo consentimiento informado. Cuantitativo: cuestionario Diabetes Attitude Scale versión 3 (DAS 3). Cualitativo: grupos focales de pacientes y entrevistas a personal para exploración de percepciones y cumplimiento sobre estilo de vida y control metabólico. Autorizado por el Comité Local de Investigación y Ética. Se utilizaron medidas de tendencia central y dispersión, frecuencias absolutas y relativas, $U$ de Mann-Whitney en DAS 3 y análisis cualitativo de respuestas en grupos focales y entrevistas. Resultados: Se incluyeron 190 pacientes diabéticos y 9 miembros del personal DiabetIMSS. En pacientes predominaron las mujeres (73\%), escolaridad primaria $(46 \%)$, edad promedio 59.6 años \pm 10.7 años, pobreza familiar baja (51.5\%), control metabólico $12.6 \%$ (intervalo de confianza [IC] del 95\%: 4.6-20.6\%) y estilo de vida desfavorable 54.6\% (IC 95\%: 41.6-67.6\%). En DAS 3 promedios menores en pacientes que en personal, con diferencias en valoración de control estricto ( $p=0.005)$. Los pacientes reconocieron la importancia de información e indicaciones para mejorar estilo de vida, y la dificultad para cumplirlas, detectaron carencias en programa, sugiriendo ca-

\section{PERCEPTION OF PATIENTS AND DIABETIMSS PROGRAM} STAFF ABOUT LIFESTYLE AND METABOLIC CONTROL

ABSTRACT: Objective: To determine reasons why neither metabolic control nor improvement on lifestyle are achieved, according to staff and patients perception in DiabetIMSS at the Unidad de Medicina Familiar (UMF) 66. Material and methods: Qualitative study from a representative sample of diabetic patients and staff in DiabetIMSS. With a previously signed consent, Diabetes Attitude Scale v. 3 (DAS 3) questionnaire was answered, focused groups of patients were formed and interviews for staff were given in order to explore their perceptions, compliance and opinions about lifestyle and metabolic control. Authorized by the Comité Local de Investigación y Ética (Local Committee of Research and Ethics). Central tendency and dispersion statistics, absolute frequencies, U of Mann-Whitney in DAS 3 and qualitative analysis were used for the answers in both, focal groups and interviews. Results: 190 diabetic patients and 9 members of the DiabetIMSS were included. Women were predominant in patients $(73 \%)$, with a basic level of education $(46 \%)$, with average age of $59.6 \pm 10.7$, low household poverty (51.5\%), metabolic control 12.63\% (CI 95\%: 4.63-20.63\%) and an unfavorable lifestyle 54.6\% (CI 95\%: 41.6-67.6\%). In DAS 3, lower averages in patients than in staff were found, with differences in strict control valuation $(p=0.005)$. Patients recognized the importance of information and advice to improve their lifestyle as difficult to achieve. They were able to detect inconsistencies and
Correspondencia:

*Ma. de Jesús Torres-Dueñas

E-mail:mjtd83@hotmail.com
Fecha de recepción: 21-01-2019

Fecha de aceptación: 05-07-2019

DOI: 10.24875/RMF.19000138 
pacitación del personal y mayores recursos. El personal reconoció incumplimiento y deserción de pacientes, limitaciones en infraestructura, capacitación, insuficiencia de personal y recursos materiales. Conclusiones: Considerar los puntos de vista emitidos por pacientes y personal para mejorar el programa y una evaluación posterior.

Palabras clave: Diabetes mellitus. Control metabólico. Estilo de vida. deficiencies, suggesting staff training and more resources. The staff admitted patient's unfulfillment and dropout, errors in infrastructure, training, and the lack of staff material resources. Conclusions: Consider points of view given by patients and staff to improve the program and subsequent evaluation. (Rev Mex Med Fam. 2019;6:95-104)

Corresponding author: Ma. de Jesús Torres-Dueñas, mjtd83@hotmail.com

Key words: Diabetes mellitus. Metabolic control. Lifestyle.

\section{INTRODUCCIÓN}

Se ha demostrado que el control intensivo de la glucemia reduce la incidencia de complicaciones microvasculares como: retinopatía, nefropatía y neuropatía en la diabetes de tipo 1 , y retinopatía y nefropatía en la diabetes de tipo $2^{1}$. Lograr el control glucémico es un reto para los pacientes y los servicios de salud $^{2}$. Una estrategia diseñada en el Instituto Mexicano del Seguro Social (IMSS) fue el programa DiabetIMSS, que inició en el año 2009 en las Unidades de Medicina Familiar (UMF) con un enfoque estructurado, integral y multidisciplinario dirigido al paciente con diabetes para la prevención de complicaciones, haciendo énfasis en el proceso educativo para modificar hábitos con el objetivo de lograr conductas positivas y un estilo de vida saludable, buscando la corresponsabilidad del paciente y su familia ${ }^{3}$.

Se han realizado numerosos estudios en diferentes partes del país a fin de determinar los alcances y limitaciones del programa DiabetIMSS, y los resultados son muy variados: mientras que en algunos casos se redujeron ciertos parámetros clínicos y bioquímicos sin alcanzar control metabólico ${ }^{4}$, o comprobaron la satisfacción del paciente en el programa aunque sin relacionarlo con el control glucémico ${ }^{5}$, en otros mejoraron los conocimientos sin lograr la capacidad de autocuidado ${ }^{6}$, o bien no lograron modificaciones en estilo de vida ${ }^{7}$, hasta llegar a notificarse investigaciones en que mejoraron tanto el control glucémico como el metabólico ${ }^{8}$.
En la UMF 66 en el periodo 2014-2016 se llevó a cabo un estudio diagnóstico para valorar el control metabólico y estilo de vida de 190 pacientes diabéticos del programa DiabetIMSS incluyendo los de nuevo ingreso, egreso inmediato y egreso tardío. Se detectó descontrol metabólico en el $87.3 \%$ y estilo de vida desfavorable en el $54.6 \%$ de los casos. Ante ello se consideró conveniente determinar la percepción de los pacientes y personal del programa DiabetIMSS sobre los motivos por los que no se logró el control metabólico ni mejoría en estilo de vida de los pacientes con diabetes mellitus (DM) en la UMF 66.

\section{MATERIAL Y MÉTODOS}

Se realizó estudio de corte mixto (cualicuantitativo) en la UMF 66 en el periodo 20162018 para dar seguimiento al estudio inicial «Control Metabólico y Estilo de Vida del programa DiabetIMSS de la UMF 66", realizado entre 2015 y 2016, que incluyó a 190 pacientes diabéticos, hombres y mujeres, participantes del programa DiabetIMSS, mayores de 18 años, quienes contaban con resultados de estilo de vida evaluados mediante el instrumento para medir Estilo de Vida en Diabético (IMEVID) y control metabólico con base en los parámetros de la American Diabetes Association (ADA) y la Norma Oficial Mexicana (NOM) NOM015-SSA2-2010. Se localizó a los pacientes, se les explicaron las características del nuevo estudio y se les solicitó su participación, previo consentimiento informado, así 
como también a los integrantes del personal del programa DiabetIMSS. De los 190 pacientes iniciales fueron 50 quienes aceptaron participar y 9 profesionales de la salud que habían colaborado en el programa durante el periodo de estudio previo también firmaron el consentimiento informado. Se eliminaron en ambos casos las encuestas incompletas.

Se utilizó el método de triangulación ${ }^{9}$ que incluyó estrategias metodológicas, tanto cualitativas como cuantitativas, para aumentar la fortaleza y calidad del estudio y procurar la validez interna ${ }^{9}$. Los resultados cuantitativos incluyeron:

1) Características sociodemográficas y familiares: tipología familiar ${ }^{10}$ (con base en el Consenso Académico en Medicina Familiar 2005) y pobreza familiar ${ }^{11,12}$ (con base en el Índice Simplificado de Pobreza Familiar ISPF, instrumento validado que integra el ingreso económico familiar, el número de hijos dependientes, la escolaridad materna y el hacinamiento para establecer 3 categorías [sin evidencia de pobreza, de 0 a 3 puntos; pobreza familiar baja, de 3.5 a 6 puntos, y pobreza familiar alta, de 6.5 a 10 puntos] $)^{11,12}$.

2) Estilo de vida, por medio del instrumento IMEVID para medir el estilo de vida en los pacientes con DM, con alfa de Cronbach global de $0.81^{13}$, constituido por 25 ítems, con siete dominios: nutrición ( $\alpha$ : 0.68 ), actividad física ( $\alpha$ : 0.53 ), consumo de tabaco ( $\alpha$ : 0.96), consumo de alcohol ( $\alpha$ : $0.90)$, información sobre diabetes ( $\alpha: 0.65$ ), emociones ( $\alpha: 0.83)$ y adherencia terapéutica ( $\alpha$ : 0.68). Donde las puntuaciones menores de 60 se consideraron como estilo de vida desfavorable, de 60 a 80 poco favorable, y mayores de 80 , favorable ${ }^{13}$.

3) Control metabólico de acuerdo con lo recomendado por la ADA y la NOM015-SSA2-2010 para la prevención, tratamiento y control de la $\mathrm{DM}^{14}$, que incluye parámetros bioquímicos como: hemoglobina glucosilada $<7 \%$, glucosa basal y preprandial $70-130 \mathrm{mg} / \mathrm{dl}$, colesterol total $<200 \mathrm{mg} / \mathrm{dl}$, triglicéridos $<150 \mathrm{mg} / \mathrm{dl}$, presión arterial $<130 / 80 \mathrm{mmHg}$, parámetros antropométricos como el índice de masa corporal $<25 \mathrm{~kg} / \mathrm{m}^{2}$, y tabaquismo negativo.

4) Actitudes y motivación sobre la diabetes, utilizando el cuestionario Diabetes Attitude Scale versión 3 (DAS 3) ${ }^{15}$, cuestionario autoaplicado con 33 ítems, integrado por 5 dimensiones (S1: necesidad de entrenamiento especial [5 preguntas]; S2: percepción de la gravedad de la DM [7 preguntas]; S3: valoración del control estricto [7 preguntas]; $\mathrm{S4}$ : valoración del impacto psicosocial de la DM (6 preguntas), y S5: autonomía del paciente [ 8 preguntas]) y un alfa de Cronbach global de 0.74, empleado para evaluar la efectividad de programas de educación diabetológica ${ }^{15}$.

A ello se sumaron dos estrategias cualitativas: una fue la integración de pacientes a Grupos Focales ${ }^{16}$ de 8 a 12 pacientes cada uno, donde se investigaron las opiniones acerca del papel de la diabetes, el estilo de vida recomendado y el logrado, el control metabólico recomendado por el equipo y el logrado, y sobre la participación del personal y el funcionamiento del programa DiabetIMSS. Se grabaron las sesiones en audio y vídeo para posteriormente transcribir la información en un editor de textos y utilizar la técnica de análisis de contenido. Cada sesión tuvo una duración de 2.5 horas. Una vez que los argumentos de los pacientes en las sesiones ya no aportaron datos nuevos a la investigación se dieron por concluidas las sesiones, con un total de cuatro sesiones. La otra estrategia cualitativa consistió en entrevistas dirigidas al personal de DiabetIMSS para establecer su opinión sobre el estilo de vida y el control metabólico recomendados en el programa y lo logrado en los pacientes, sobre el cumplimiento de los pacientes al programa y el funcionamiento de este, grabándose en audio para posteriormente transcribir la información y analizarla. De esta manera se procuró visualizar el problema desde diferentes ángulos para aumentar la validez y consistencia de los resultados, ampliando la perspectiva para 
interpretar el fenómeno a estudiar y procurar enriquecer el estudio ${ }^{16}$.

El proyecto fue valorado en el Comité Local de Investigación en Salud y aprobado por el Sistema de Registro Electrónico en Línea de la Coordinación de Investigación en Salud (SIRELCIS) del IMSS con número de registro R-2017-3004-7.

\section{ANÁLISIS ESTADÍSTICO}

Para el análisis cuantitativo se analizaron las características sociodemográficas, el control metabólico y el estilo de vida de los pacientes, posteriormente los resultados del cuestionario DAS 3, que mostró los promedios tanto de los pacientes como del personal de salud en los cinco rubros que evalúa, comparándose mediante la prueba no paramétrica $U$ de Mann-Whitney, dado que no fue posible establecer la normalidad de los datos reportados. Se utilizó un nivel de confianza del 95\%.

Para el análisis cualitativo de la información se trascribieron los audios de las sesiones agrupando los resultados en categorías, considerando los objetivos específicos sobre control metabólico y estilo de vida, opinión sobre el programa DiabetIMSS y sugerencias a este. Igualmente se transcribieron los audios de las entrevistas al personal de DiabetIMSS considerando los mismos puntos y procurando respetar el discurso de los participantes en todos los rubros.

\section{RESULTADOS}

En relación con las características sociodemográficas, predominaron las mujeres, con escolaridad primaria, los casados y dedicados a labores del hogar, pertenecientes a familias nucleares simples, de núcleo integrado y con pobreza familiar baja (Tabla 1).

Sobre las variables bioquímicas, en conjunto, de acuerdo con criterios de ADA y NOM solo el $12.6 \%$ de los pacientes se encontraban controlados metabólicamente. Tanto en los pacientes de nuevo ingreso (61), egreso reciente (42) o egreso tardío (87) se observaron promedios por arriba del control metabólico en hemoglobina glucosilada, colesterol total y triglicéridos, y el índice de masa corporal se mantuvo alto en todos los grupos. Solo hubo diferencias estadísticamente significativas en las cifras de glucemia de acuerdo con el tiempo de ingreso al programa (Tabla 2).

En actitudes y motivaciones sobre diabetes en los pacientes y en el personal de salud se observaron promedios más bajos en los primeros en todas las subescalas, aunque únicamente hubo diferencias estadísticamente significativas en el rubro de valoración del control estricto (Fig. 1).

Las percepciones de los pacientes sobre control metabólico, estilo de vida y sobre el programa DiabetIMSS se resumen a grandes rasgos en la tabla 3 y en cuanto a lo observado por el personal sobre los mismos rubros, se encuentran en la tabla 4.

\section{DISCUSIÓN}

En nuestra investigación pudimos observar las coincidencias de las características sociodemográficas de nuestros pacientes con estudios previos: la participación mayoritaria de mujeres ${ }^{1,2,4,6,8,17,18}$, la edad ${ }^{1,2,4,6,8,17,18}$ y la escolaridad primaria ${ }^{1,2,6,17,18}$, todo ello va en relación con la mayor asistencia de mujeres a nuestras clínicas y que es el grupo de 60 años y más el prevalente en los diabéticos. Tuvimos un mayor porcentaje de casados $(54 \%)^{2,5,8}$ y más pacientes dedicadas a labores del hogar ${ }^{1,2,5,8,18}$. Sobre las características familiares de los participantes, son escasas las investigaciones que lo consignan, sin embargo en nuestro caso la pertenencia a familias con núcleo integrado y pobreza familiar baja fue semejante a lo reportado por Mendoza ${ }^{18}$.

En parámetros bioquímicos, nuestros grupos de estudio reportaron cifras de hemoglobina glucosilada, glucosa promedio y lípidos por arriba de los valores de control, al igual que lo observado por Barceló ${ }^{1}$, Leyva $^{6}$, Romero ${ }^{7}$ y González ${ }^{19}$, únicamente Ortega $^{4}$ y Figueroa ${ }^{8}$ en su investigación reportan valores normales.

El porcentaje de pacientes controlados de nuestro estudio (12.6\%) considerando 
Tabla 1. Características sociodemográficas de pacientes del programa DiabetIMSS UMF 66

\begin{tabular}{|c|c|c|c|}
\hline \multicolumn{2}{|c|}{ Características Sociodemográficas } & \multirow{2}{*}{$\begin{array}{c}\begin{array}{c}\text { Frecuencia } \\
(\mathbf{n}=\mathbf{1 9 0})\end{array} \\
138\end{array}$} & \multirow{2}{*}{$\begin{array}{c}\text { Porcentaje } \\
(\%)\end{array}$} \\
\hline Género & Femenino & & \\
\hline & Masculino & 52 & 27 \\
\hline Edad* & 59.6 años \pm 10.7 & & \\
\hline \multirow[t]{8}{*}{ Escolaridad } & Primaria & 87 & 46 \\
\hline & Secundaria & 35 & 18 \\
\hline & Analfabeta & 26 & 14 \\
\hline & Licenciatura & 20 & 11 \\
\hline & Preparatoria & 13 & 7 \\
\hline & Técnico & 6 & 3 \\
\hline & Sin datos & 3 & 1 \\
\hline & Total: & 190 & 100 \\
\hline \multirow[t]{7}{*}{ Estado civil } & Casado & 103 & 54 \\
\hline & Soltero & 36 & 19 \\
\hline & Viudo & 20 & 11 \\
\hline & Divorciado & 12 & 6 \\
\hline & Unión Libre & 10 & 9 \\
\hline & Sin datos & 1 & 1 \\
\hline & Total: & 190 & 100 \\
\hline \multirow[t]{9}{*}{ Ocupación } & Hogar & 147 & 77 \\
\hline & Comerciante & 13 & 7 \\
\hline & Profesional & 12 & 6 \\
\hline & Sin datos & 6 & 3 \\
\hline & Desempleado & 4 & 2 \\
\hline & Agricultura / Ganadería & 3 & 2 \\
\hline & Técnica & 3 & 2 \\
\hline & Artesano & 2 & 1 \\
\hline & Total: & 190 & 100 \\
\hline \multirow[t]{2}{*}{ Tipología familiar ${ }^{\dagger}$} & Núcleo Integrado & 99 & 52 \\
\hline & Nuclear Simple & 48 & 25 \\
\hline Medios de subsistencia $^{\dagger}$ & Servicios & 154 & 81 \\
\hline Pobreza familiar ${ }^{\dagger}$ & Baja & 98 & 51.5 \\
\hline
\end{tabular}

*Promedio $\pm D E$

†Solo se anotan las categoría mas frecuentes

los parámetros de ADA y NOM (hemoglobina glucosilada, glucosa, colesterol, triglicéridos e índice de masa corporal) fue mucho menor que lo observado en otras investigaciones $\left(46.5^{19}, 48^{1}, 58.5^{6} \mathrm{y}\right.$ $\left.70.4 \%{ }^{8}\right)$. El estilo de vida desfavorable en nuestros pacientes (54.6\%) fue semejante a lo observado por $\operatorname{Urban}^{17}(67.9 \%)$ y González $^{19}(55.2 \%)$, que contrasta totalmente con lo reportado por Figueroa ${ }^{8}$, quien encontró en su investigación que el $84.6 \%$ de los pacientes egresados de 
Tabla 2. Control metabólico y estilo de vida de pacientes del Programa DiabetIMSS, Unidad Médica Familiar n. ${ }^{\circ} 66$

\begin{tabular}{|c|c|c|c|c|}
\hline $\begin{array}{l}\text { Parámetros } \\
\text { bioquímicos** }\end{array}$ & $\begin{array}{c}\text { Nuevo ingreso } \\
(1-6 \text { meses }) \\
(n=61)\end{array}$ & $\begin{array}{c}\text { Egreso reciente } \\
(12-18 \text { meses }) \\
(\mathrm{n}=42)\end{array}$ & $\begin{array}{c}\text { Egreso tardío } \\
\begin{array}{c}(>18 \text { meses }) \\
(n=87)\end{array}\end{array}$ & $\begin{array}{l}\text { Valor } \\
\text { dep }\end{array}$ \\
\hline \multicolumn{5}{|c|}{ Control metabólico } \\
\hline $\mathrm{HbA1} \mathrm{c}^{*}$ & 7.72 & 7.56 & 7.66 & 0.91 \\
\hline $\begin{array}{l}\text { Glucemia basal* } \\
\mathrm{mg} / \mathrm{dl}\end{array}$ & 132.64 & 153.05 & 157.06 & 0.02 \\
\hline $\begin{array}{l}\text { Colesterol total* } \\
\mathrm{mg} / \mathrm{dl}\end{array}$ & 198.79 & 204.71 & 209.17 & 0.25 \\
\hline $\begin{array}{l}\text { Triglicéridos* } \\
\mathrm{mg} / \mathrm{dl}\end{array}$ & 221.97 & 224.95 & 219.44 & 0.87 \\
\hline $\begin{array}{l}\text { Índice de masa corporal* } \\
\mathrm{kg} / \mathrm{m}^{2}\end{array}$ & 30.82 & 32.42 & 30.53 & 0.32 \\
\hline \multicolumn{5}{|c|}{ Estilo de vida } \\
\hline Desfavorable $^{\dagger}$ & 53 & 58 & 53 & 0.87 \\
\hline Poco favorable ${ }^{\dagger}$ & 34.6 & 32.6 & 35.4 & 0.50 \\
\hline Favorable $^{\dagger}$ & 12.4 & 9.4 & 11.6 & 0.32 \\
\hline
\end{tabular}

*Promedios.

'Porcentajes.

HbA1c: hemoglobina glucosilada.

DiabetIMSS contaban con un estilo de vida favorable.

Al aplicar el cuestionario DAS 3 en todas las subescalas fueron menores los valores promedios de los pacientes respecto al personal con diferencias estadísticamente significativas en la valoración de control estricto, similar a lo reportado por Mendoza ${ }^{18}$. Probablemente esto se explique debido a que el personal enfoca sus esfuerzos en este aspecto, de manera que se convierte en el objetivo principal en el manejo del paciente ${ }^{18}$.

\section{Resultados cualitativos en los pacientes}

\section{Percepción sobre diabetes}

Los pacientes consideraron a la diabetes como una enfermedad debilitante que les ocasionaba bajo estado de ánimo, limitaciones para realizar sus actividades cotidianas y percepción de que son una carga para la familia, con preocupación acerca de las complicaciones de la diabetes; y en aquellos que ya presentaban complicaciones crónicas, estas limitaban sus actividades de la vida diaria, su nivel de independencia para actividades básicas e instrumentadas y mermaban su calidad de vida. Todo ello coincide con Moraga ${ }^{20} \mathrm{y}$ con Mendoza Vázquez ${ }^{21}$.

\section{Estilo de vida}

La mayoría de los pacientes mencionaron que durante su estancia en el programa DiabetIMSS recibieron la información suficiente para modificar sus hábitos de alimentación e intentaron apegarse a las indicaciones recibidas, pero les fue difícil realizarlos por circunstancias personales o por falta de apoyo de su familia, cuestión ya consignada por Mendoza Vázquez ${ }^{21}$, que cita: «por lo común la gente próxima al diabético no se solidariza con él». Opinaron que la información nutricional no fue individualizada, emitiendo conceptos poco claros sobre los alimentos, coincidente con lo detectado por Ortega ${ }^{4}$, quien reportó que el 65.9\% de sus pacientes percibieron atención nutricional inadecuada. Asimismo, los pacientes consideraron difícil llevar a cabo la dieta 


\section{DAS 3}

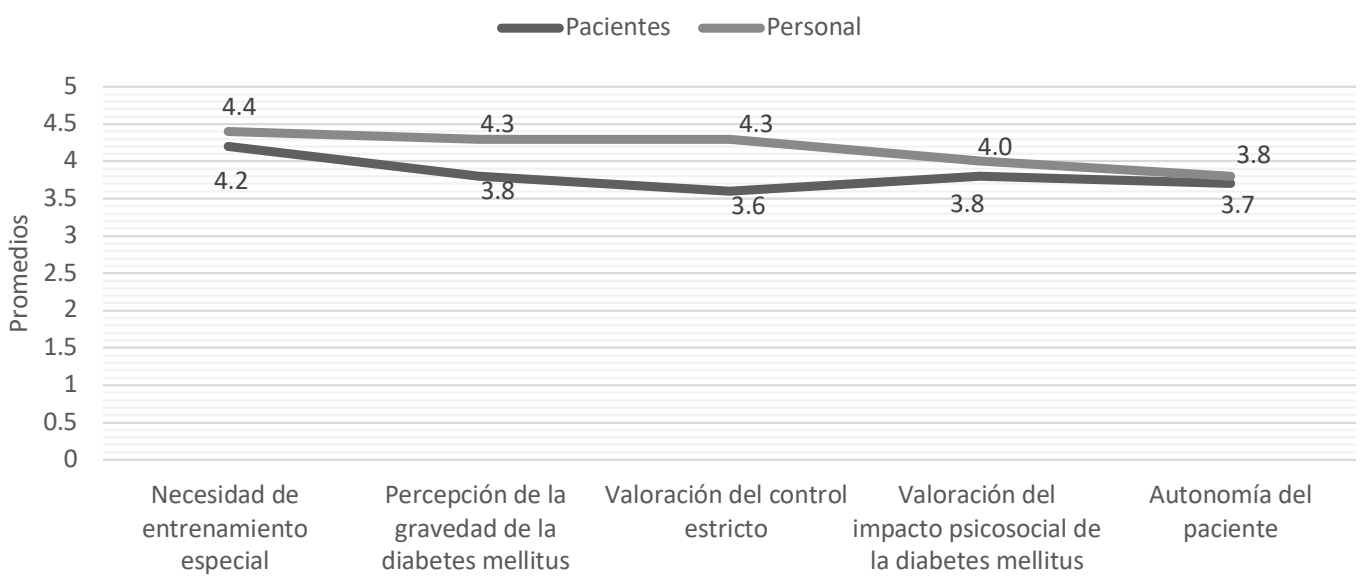

$p=0.0055$ (valoración del control estricto).

Figura 1. Promedios en subescalas de Actitudes y Motivaciones en Diabetes entre pacientes y personal de salud del Programa DiabetIMSS de la Unidad Médica Familiar n. ${ }^{0} 66$.

indicada por problemas económicos, gustos, preferencias y costumbres, lo cual también fue reportado por Mendoza Vázquez ${ }^{21}$. Por el contrario, Barceló ${ }^{1}$ atribuyó el control metabólico del $65 \%$ de sus pacientes al seguimiento de su dieta. Sobre la actividad física durante el programa, fue poco realizada e incluso negaron haber recibido capacitación, aduciendo además falta de tiempo y de áreas adecuadas para su práctica.

\section{Control metabólico}

Los pacientes fueron informados acerca de sus resultados de glucosa en ayuno como parte del monitoreo sobre su control y algunos mencionaron que sí disminuyeron sus cifras de glucosa, pero sin llegar a los valores de control.

\section{Percepción sobre DiabetIMSS}

Consideraron a DiabetIMSS un buen programa, donde les informaron sobre la diabetes y sus complicaciones, sobre los estilos de vida favorables en alimentación y ejercicio, semejante a lo reportado por Morales ${ }^{22}$, sin embargo opinaron que se debe mejorar la temática de las sesiones educativas, contar con mayores recursos didácticos, mejorar la información nutricional, y organizar los tiempos entre consulta y sesiones educativas, ya que se interrumpen, con tiempos reducidos para la consulta, coincidiendo con Morales $^{22}$, donde el factor tiempo fue el principal limitante. Los pacientes solicitaron proveer de personal suficiente de Psicología, Estomatología y Nutrición, y monitores para actividad física, igual que lo reportado por Rodríguez ${ }^{23}$ y Zúñiga ${ }^{24}$. Así también mejorar los espacios físicos, pues son reducidos y procurar mayor limpieza de las áreas, semejante a lo detectado por Morales ${ }^{22}$.

\section{Resultados cualitativos en el personal}

\section{Estilo de vida}

El personal consideró que aunque el paciente se dice informado acerca de modificar los estilos de vida desfavorables, no lo llevan a cabo, hecho similar a lo reportado por Velasco ${ }^{25}$, quien menciona que al tratarse de una enfermedad crónica de inicio y evolución gradual, no se percibe por el paciente como grave, por lo que la adherencia al tratamiento disminuye considerablemente en comparación a otros padecimientos.

\section{Controlmetabólico}

El personal consideró que los pacientes no logran control debido a que no se apegan a las indicaciones médicas, al abandono del 
Tabla 3. Percepción de los pacientes sobre control metabólico, estilo de vida y Programa DiabetIMSS

\begin{tabular}{|c|c|c|}
\hline Control metabólico & Estilo de vida & Opinión DiabetIMSS \\
\hline $\begin{array}{l}\text { "... de hecho (la glucosa) nunca se } \\
\text { controló, siempre estuvo allí,... 170, lo } \\
\text { más que llegaba a bajarla 160...» }\end{array}$ & $\begin{array}{l}\text { "... mi problema es que yo no puedo } \\
\text { dejarlo (refresco de cola). Yo no puedo } \\
\text { desayunar sin mi vaso de...» }\end{array}$ & $\begin{array}{l}\text { «Se necesita mayor capacidad de las perso- } \\
\text { nas que están enfrente de un diabético como } \\
\text { yo, me dicen algo y yo ya me pongo o a la } \\
\text { defensiva o agresivo o me pongo a llorar.» }\end{array}$ \\
\hline $\begin{array}{l}\text { "El primer año síme controlé, pero el } \\
\text { segundo año tenía muchas cosas emocio- } \\
\text { nales... entonces todo eso hace que a mí } \\
\text { me suba.» }\end{array}$ & $\begin{array}{l}\text { «L verdad, yo no llevo la dieta exacta- } \\
\text { mente como me la da la doctora porque } \\
\text { es demasiado.» }\end{array}$ & $\begin{array}{l}\text { «Falta tiempo para la consulta. . . siem- } \\
\text { pre me quedé con dudas respecto a mi } \\
\text { tratamiento.» }\end{array}$ \\
\hline $\begin{array}{l}\text { "Actualmente tengo } 130 \text { o } 140 \text { y trato } \\
\text { de bajarla más, pero sí, me ha costado la } \\
\text { verdad, bastante...» }\end{array}$ & $\begin{array}{l}\text { «Hay que caminar todos los días, } \\
\text { cuando menos nos dijeron media hora, } \\
20 \text { minutos, pero hay que caminar. ¿Lo } \\
\text { hacemos? No lo hacemos.» }\end{array}$ & $\begin{array}{l}\text { "... Io pesaban a uno allá abajo y pesaba } \\
60,10 \text { pesaban aquí } 62, \text { lo pesaban aden- } \\
\text { tro } 70, \text { no, no, la verdad, esos detalles..." }\end{array}$ \\
\hline $\begin{array}{l}\text { "... a mí nunca se me llegó a controlar, } \\
\text { incluso a la fecha manejo valores altos.» }\end{array}$ & $\begin{array}{l}\text { «Cada vez que me pico digo... hijole ien } \\
\text { qué momento no hice caso cuando me } \\
\text { dijeron baja de peso, ... no somos obe- } \\
\text { dientes con el médico, somos necios.» }\end{array}$ & $\begin{array}{l}\text { "Faltan recursos... como un aula más } \\
\text { grande...» } \\
\text { "... que las pláticas fueran más dinámicas } \\
\text { e incluyentes, que te sientas importante.» }\end{array}$ \\
\hline
\end{tabular}

Tabla 4. Percepción del personal sobre cumplimiento de los pacientes al Programa DiabetIMSS

\begin{tabular}{|l|l|l|}
\hline Control metabólico & Estilo de vida & Opinión DiabetIMSS \\
\hline $\begin{array}{l}\text { "Los pacientes no se apegan a las indi- } \\
\text { caciones médicas, por lo que no logran el } \\
\text { control metabólico.» }\end{array}$ & $\begin{array}{l}\text { "El paciente no pone de su parte para } \\
\text { mejorar, no realizan las actividades } \\
\text { sugeridas.» }\end{array}$ & $\begin{array}{l}\text { Capacitación al personal } \\
\text { «No se cuenta con el personal que marca } \\
\text { el manual, prácticamente solo con médico } \\
\text { yenfermera...» }\end{array}$ \\
\hline $\begin{array}{l}\text { «El paciente sabe que al cuidarse solo } \\
\text { uno o días antes de su estudio de glucosa } \\
\text { de ayuno va a salir bien.» }\end{array}$ & $\begin{array}{l}\text { «Ausentismo y abandono del programa.» } \\
\text { "Cumplimiento al programa de un 10 a } \\
\text { un 50\%.» }\end{array}$ & «Falta de tiempo para la consulta...» \\
\hline «Falta de motivación y autoestima.» & $\begin{array}{l}\text { "El nivel educativo y las costumbres } \\
\text { que tiene el paciente influye sobre los } \\
\text { alimentos y su preparación.» }\end{array}$ & $\begin{array}{l}\text { Pacientes mal referidos al programa } \\
\text { Falta de espacio adecuado, recursos } \\
\text { didácticos y glucómetro }\end{array}$ \\
\hline $\begin{array}{l}\text { «Muchos deciden que pueden vivir sin } \\
\text { necesidad de tanto cuidado o se re- } \\
\text { húsan... y entonces es donde empiezan } \\
\text { a abandonar...» }\end{array}$ & $\begin{array}{l}\text { "Falta de apoyo por parte de su familia } \\
\text { para apegarse a la dieta.» } \\
\text { "Se sienten una carga para su familia.» }\end{array}$ & $\begin{array}{l}\text { Restricciones en laboratorio: hemoglobi- } \\
\text { na glucosilada y perfil de lipidos completo } \\
\text { Restricciones en medicamentos: insulina } \\
\text { glargina e hipoglucemiantes orales (de } \\
\text { transcripción) }\end{array}$ \\
\hline
\end{tabular}

programa, a la falta de motivación personal y de conocimiento sobre las complicaciones de la diabetes. Como menciona Mendoza ${ }^{18}$ : "el conocimiento de las complicaciones de la diabetes no es suficiente para modificar patrones de vida» pues aunque los pacientes reciban la información encuentran difícil adherirse a esas actitudes positivas a su vida ${ }^{18}$.

Coincidimos con Rodríguez ${ }^{23}$ en que el programa de DiabetIMSS ha mostrado tener impactos positivos en el manejo y control de la diabetes, sin embargo, requiere de algunas modificaciones en su contenido y estructura a fin de optimizar los resultados no solo en el enfermo, sino también en la familia y en el colectivo ${ }^{23}$.

\section{Percepción sobre DiabetIMSS}

Fue generalizada la opinión de la carencia de personal de Psicología, Estomatología y Nutrición, y el no contar con médicos y enfermeras suplentes, coincidiendo con 
Leyva $^{6}$ y Zúñiga ${ }^{24}$, donde la falta de equipo multidisciplinario propio para el módulo limita las acciones para la adopción de estilos de vida saludables ${ }^{17,19,22-24}$. Doubova ${ }^{25}$ refiere que el programa requiere aumentar la plantilla de médicos (1.2 veces), de nutriólogos (4.2 veces) y de trabajadores sociales (4.1 veces). Por otro lado, en nuestro estudio el personal negó recibir algún tipo de capacitación al integrarse al equipo y solo haberle entregado el Manual del Módulo DiabetIMSS, acción insuficiente para adquirir la experiencia necesaria en la práctica del modelo educativo, situación observada por Leyva ${ }^{6}$, quien considera primordial que los educadores tengan una capacitación especializada y certificada sobre la educación y el tratamiento del paciente diabético. En relación al Manual Operativo del Programa, coincidimos con Morales ${ }^{22}$ en que es necesaria una revisión, pues la última actualización data del año 2009. Entre otras carencias mencionadas por el personal estuvieron la hemoglobina glucosilada y el perfil de lípidos completo, coincidiendo a lo reportado por $\mathrm{Gil}^{26}$, la falta de glucómetro y la necesidad de una orientación adecuada en las supervisiones para llevar a cabo las tareas que indica el manual, pues como lo expresó un miembro del personal, «nunca supe si llevamos adecuadamente el programa», situación ya observada por Velasco ${ }^{27}$, que reporta que el programa de DiabetIMSS se evalúa constantemente por el mismo personal, pero la inconsistencia en la información, la falta de recursos y la ejecución parcial del programa impiden que se tenga un diagnóstico fiable de las áreas de oportunidad, lo cual repercute en su eficacia y permanencia, pues la mayoría de los pacientes no tienen control metabólico ${ }^{2,6,19,23}$. El personal médico también mencionó tiempo insuficiente destinado a la consulta, semejante a lo reportado por Doubova ${ }^{25}$, pues el tiempo promedio requerido para atender a los pacientes diabéticos debería ser de: 35 minutos de consulta médica, 25 minutos de enfermería, y 40 minutos de nutrición y trabajo social, tiempos que en nuestra unidad no se cumplen.

\section{CONCLUSIONES}

El descontrol metabólico y el inadecuado estilo de vida fueron el punto de inicio para darle voz a los actores involucrados: pacientes y personal del programa DiabetIMSS. Fue así que los primeros reconocieron haber recibido la información necesaria en el programa para modificar su estilo de vida, sin embargo hubo dificultades para realizarlo, detectaron irregularidades y carencias en el programa, sugiriendo capacitación del personal y mayores recursos. Por lo que toca al personal de salud, por una parte reconoció incumplimiento y deserción de los pacientes, y por otra, errores en infraestructura, capacitación, insuficiencia de personal y recursos materiales.

Será importante considerar los puntos de vista emitidos por pacientes y personal para realizar los cambios necesarios a fin de lograr a futuro un mayor cumplimiento y autocuidado que se traduzca en un mejor control de los pacientes y una mayor satisfacción del personal, y una vez efectuados los cambios emprender una nueva evaluación.

Respecto a las limitaciones del estudio, los resultados pudieran considerarse no generalizables a otros contextos, en virtud de que son situaciones específicas del ámbito estudiado, sin embargo son muchas las similitudes existentes ya documentadas en otras investigaciones.

\section{CONFLICTO DE INTERESES}

Los autores declaran no tener ningún conflicto de interés.

\section{BIBLIOGRAFÍA}

1. Barceló A, Robles $S$, White $F$, Jadue L, Vega J. Una intervención para mejorar el control de la diabetes en Chile. Rev Panam Salud Publica/Pan Am J Public Health. 2001;10(5):328-33.

2. León Mazón MA, Araujo Mendoza GJ, Linos Vázquez ZZ. DiabetIMSS. Eficacia del programa de educación en diabetes en los parámetros clínicos y bioquímicos. Rev Med Inst Mex Seguro Soc. 2012;51(1):74-9.

3. Guía de Práctica Clínica. Diagnóstico, metas de control ambulatorio y referencia oportuna de prediabetes y diabetes mellitus tipo 2 en adultos en el primer nivel de atención [Internet] México: Secretaría de Salud; 2013. Disponible en: http://www. cenetec.salud.gob.mx/descargas/gpc/CatalogoMaestro/093_ GPC_Diabmellitus2/SSA_093_08_GRR.pdf 
4. Ortega Morán C, Calderón González MR, Gómez Alonso C, Muñoz Cortés G. Satisfacción de pacientes del Módulo DiabetIMSS y su asociación al control glucémico en una unidad de medicina familiar. Aten Fam. 2017;24(2):77-81.

5. Franco Calderón MS. Evaluación de la satisfacción del paciente diabético tipo 2 adscrito en el programa DiabetIMSS de la UMF 1 delegación Aguascalientes [tesis]. Aguascalientes: Universidad Autónoma de Aguascalientes; 2015

6. Leyva Jiménez R, Torres González G, Pérez Arroyo M A, Maya Juárez A. Educación diabetológica en la atención primaria. Salud y Ciencia. 2014;20(7):720-5

7. Romero Valenzuela E, Zonana Nacach A, Colín García MA Control de glucosa en pacientes que asistieron al programa de educación DiabetIMSS en Tecate, Baja California. Med Int Mex. 2014;30(5):554-61.

8. Figueroa Suárez ME, Cruz Toledo JE, Ortiz Aguirre AR, Lagunés Espinosa AL, Jiménez Luna J, Rodríguez Moctezuma JR. Estilo de vida y control metabólico en diabéticos del programa DiabetIMSS. Gac Med Mex. 2014;150(1):29-34.

9. Okuda Benavides M, Gómez-Restrepo C. Métodos en investigación cualitativa: triangulación. Rev Colomb Psiquiatr. 2005:34(1):118-24

10. Consenso Académico en Medicina Familiar de Organismos e Instituciones Educativas y de Salud (PRCAMF). Conceptos básicos para el estudio de las familias. Arch Med Fam. 2005;7(1):15-9.

11. Ponce-Rosas ER, Irigoyen-Coria A, Gómez-Clavelina F, TeránTrillo M, Madrigal DH, Palomino GL. Confiabilidad del índice simplificado de marginación familiar. Arch Med Fam. 1999;1:99-104.

12. Ponce-Rosas $R E$, Irigoyen-Coria A, Terán-Trillo M, Gómez Clavelina F, Landgrave-lbáñez S, Mosqueda-Pérez G. Validez de constructo del índice simplificado de pobreza familiar (ISPF). Arch Med Fam. 2002;4:6-12.

13. López Carmona JM, Ariza Andraca CR, Rodríguez Moctezuma JR Munguía Miranda C. Construcción y validación inicial de un instrumento para medir el estilo de vida en pacientes con diabetes mellitus tipo 2. Salud Pub Mex. 2003;45(4):259-68.

14. Norma Oficial Mexicana NOM-015-SSA2-2010. Para la prevención tratamiento y control de la diabetes mellitus [Internet]. México: Diario Oficial de la Federación. 23 junio 2009. Disponible en: http://www.dof.gob.mx/normasOficiales/4215/salud/salud.htm

15. Hernández Anguera JM, Basora Gallisa J, Millán Guasch M Figueroa Pino D. La versión española de la Diabetes Escala de Actitud (DAS-3sp): un instrumento de medición de actitudes y motivaciones en diabetes. Endocrinol Nutr. 2002;9(9):293-8.
16. Hamui-Sutton A, Varela-Ruiz M. La técnica de grupos focales. Inv Ed Med. 2013;2(1):55-60.

17. Urbán-Reyes BR Coghlan-López Jل Jastañeda-Sánchez O. Estilo de vida y control glucémico en pacientes con diabetes mellitus en el primer nivel de atención. Aten Fam. 2015;22(3):68-71.

18. Mendoza Rivera R, Gil Alfaro I, Soler Huerta E, Mendoza Sánchez $\mathrm{H}$, Hernández Anguera J. Actitudes y motivaciones del paciente diabético y el personal de salud sobre la diabetes mellitus tipo 2. Arch Med Fam. 2009;11(3):93-8.

19. González Hernández L, Albavera Hernández $C$, León Zeompoalteca T, Román Velázquez M. Estilo de vida y control glucémico en pacientes diabéticos del programa DiabetIMSS en Cuernavaca, Morelos. Rev Mex Med Fam. 2017;4(1):4-8.

20. Moraga PR. Estudio cualitativo sobre la experiencia subjetiva de la enfermedad en diabéticos. Rev Chil Salud Publica. 2005;9(3):162-8

21. Mendoza Vázquez F, Velázquez Monroy O, Martín MA, Pego Pratt U, Campero Marín EC, Lara EA, et al. Comunicación y perspectiva de audiencias: una investigación cualitativa para la diabetes. Rev Endocrinol Nutr. 2000;8(1):5-13.

22. Morales Pérez Ml, Urbina Aguilar BA, Zavala Cruz GG, Rodríguez Pérez CV. Estudio cualitativo del programa DiabetIMSS en una Unidad de Medicina Familiar de San Luis Potosí. Rev Enferm Inst Mex Seguro Soc. 2017;25(2):101-10.

23. Rodríguez Torres A, Casa Patiño D, Contreras Landgrave G, Camacho Ruíz JE, Galicia Andrade A. Análisis de un programa médico asistencial para diabetes (DiabetIMSS) mediante el método FODA. Revista médica de la Universidad de Costa Rica. 2015;9(1):55-65.

24. Zúñiga Ramírez MG, Villareal Ríos ER, Galicia Rodríguez L, Martínez González L, Cervantes Becerra RG. Perfil de uso de los servicios del módulo DiabetIMSS por pacientes con diabetes mellitus 2. Rev Enferm Inst Mex Seguro Soc. 2013;21(2):79-84.

25. Doubova SV, Ramírez-Sánchez C, Figueroa-Lara A, Pérez-Cuevas R. Recursos humanos para la atención de pacientes con diabetes en unidades de medicina familiar del Instituto Mexicano del Seguro Social. Salud Publica Mex. 2013;55:607-17.

26. Gil Velázquez LE, Sil Acosta MJ, Aguilar Sánchez L, Echeverría Zuno S, Michaus Romero F, et al. Perspectiva de la diabetes mellitus tipo 2 en el Instituto Mexicano del Seguro Social. Rev Med Inst Mex Seguro Soc. 2013;51(1):58-67.

27. Velasco Fuentes MC. Diseño y evaluación de un programa centrado en la adherencia terapéutica en diabetes mellitus Tipo 2 [tesis]. Toluca, México: Universidad Autónoma del Estado de México; 2016. 